\title{
Atlas of sentinel lymph nodes in early breast cancer using single-photon emission computed tomography: implication for lymphatic contouring
}

\author{
Sergey Nikolaevich Novikov, Pavel Ivanovich Krzhivitskii, Yulia Sergeevna Melnik, Alina Albertovna Valitova, \\ Zhanna Viktorovna Bryantseva, Irina Alexandrovna Akulova, Sergey Vasilevich Kanaev \\ Department of Radiation Oncology \& Nuclear Medicine, N.N. Petrov National Medical Research Center of Oncology, St. Petersburg, Russia
}

Received: October 16, 2020

Revised: February 2, 2021

Accepted: February 3, 2021

\section{Correspondence:}

Sergey Nikolaevich Novikov Department of Radiation Oncology \& Nuclear Medicine, N.N. Petrov

National Medical Research Center of Oncology, St. Petersburg 197758,

Russia

Tel: +7-9500437996

E-mail:krokon@mail.ru

ORCID:

https://orcid.org/0000-0002-7185-1967
Purpose: To determine the localization of sentinel lymph nodes (SLNs) in a large cohort of patients with breast cancer and validate the European Society for Therapeutic Radiology and Oncology (ESTRO), Radiation Therapy Oncology Group (RTOG), and Radiotherapy Comparative Effectiveness (RADCOMP) guidelines on regional lymph node clinical target volume (CTV-LN) delineation.

Materials and Methods: A total of 254 women with cT1-3N0-1M0 breast cancer underwent single-photon emission computed tomography (SPECT-CT) visualization of SLNs after intra- and peritumoral injection of 99mTc-radiocolloids. All SPECT-CT images were fused with reference simulation computed tomography. A 3D atlas of SLNs was created and used for evaluation of CTV-LN defined by contouring guidelines.

Results: SPECT-CT visualized 532 SLNs that were localized in axillary level I in $67.5 \%$, level II in $15.4 \%$, level III in 7.3\%, internal mammary in $8.5 \%$, and supraclavicular in $1.3 \%$ cases. The majority of level II-IV and internal mammary SLNs were inside the recommended CTV-LN. Axillary level I SLNs were covered by ESTRO and RTOG contours in 85\% and 85\% cases, respectively. "Out of contours" SLNs were mostly detected in lateral subgroup of level I LN (18.5\%), while 98\%-99\% of anterior pectoral and central axillary SLNs were covered by CTV-LN. Internal mammary SLNs were visualized in 33 cases and were outside ESTRO and RTOG contours in 3 and 6 observations, respectively. Conclusion: SPECT-CT atlas of SLNs demonstrated that in most cases ESTRO and RTOG guidelines correctly represented CTV-LNs with the exception of lateral subgroup of SLNs.

Keywords: Breast cancer, Lymph nodes, Target volume delineation, Sentinel lymph nodes

\section{Introduction}

The concept of sentinel lymph nodes (SLNs) was proposed in the last quarter of the 20th century and was extensively evaluated in patients with melanoma and breast cancer [1]. The concept postulated a predictive and sequential fashion of regional lymph node (LN) involvement with the regional spread predominantly to the LNs that were the first on the drainage way from the primary lesion (these LNs were called sentinel); subsequent invasion of the second echelon LNs that received lymph fluid from the sentinel nodes; and finally with a regional dissemination of the tumour to other regional LNs. This concept was successfully validated in prospective multicentre trials and retrospective meta-analysis and now is widely accepted in clinical practice [1-3]. Mapping of SLNs by radiocolloids becomes the basic technique that permits a precise localization of SLNs and a subsequent radioguided surgery. Taking into account the sequential order of regional LNs involvement and prevalence of SLNs invasion by the tumour, we propose that the

Copyright (C) 2021 The Korean Society for Radiation Oncology

This is an Open Access article distributed under the terms of the Creative Commons Attribution Non-Commercial License (http://creativecommons.org/licenses/by-nc/4.0/) which permits unrestricted non-commercial use, distribution, and reproduction in any medium, provided the original work is properly cited. 
atlas of SLNs can be an effective tool that would help to validate the most widely used European Society for Therapeutic Radiology and Oncology (ESTRO) and Radiation Therapy Oncology Group (RTOG) guidelines for contouring regional LNs in patients with breast cancer. We expect that this atlas can be especially useful as a tool for a precise definition of LN clinical target volume (LN-CTV) in women with an early breast cancer. First of all, it can be important for LN contouring in women with $\mathrm{N} 1$ breast cancer and patients with positive SLNs that would receive postoperative LNs irradiation instead of an axillary LNs dissection.

The primary aim of the this study was to create a 3D atlas of SLNs in patients with early breast cancer using single-photon emission computed tomography (SPECT-CT) and evaluate the validity of ESTRO, RTOG, and RADCOMP (Radiotherapy Comparative Effectiveness) Consortium Trial contouring guidelines [4-6].

\section{Materials and Methods}

This study was reviewed by review board and Ethical Committee of the N.N. Petrov National Medical Research Center of Oncology (No. 108). A total 254 women with an early (T1-3NOM0) breast cancer underwent SPECT-CT visualization of SLNs before a routine SLN biopsy between February 2014 and April 2020. The SPECT-CT examination started 60-120 minutes after intra- and peritumoral injection of $100-150 \mathrm{MBq}$ of $99 \mathrm{mTc}$-radiocolloids with particle size of $80-1,000 \mathrm{~nm}$. The acquisition was performed in position with arms $140^{\circ}-170^{\circ}$ abducted above the head. The anatomical position of SLNs on SPECT-CT images was allocated to the following axillary subgroups that had been previously described in details [7]: anterior pectoral, central and lateral level I LNs, subscapular, interpectoral, subpectoral level II LNs, apical level III LNs. In addition, supraclavicular and internal mammary LNs were considered as regional for breast cancer patients. All SLNs were contoured on every SPECT-CT data set and color-coded according to their allocation to different groups and subgroups of the regional LNs. Finally we obtained images of 135 women with left (289 SLNs) and 243 (243 SLNs) with right breast cancer.

For the reference CT image we used a simulation CT scan of a female patient $(164 \mathrm{~cm}, 60 \mathrm{~kg})$ who had undergone a left-sided breast conserving surgery and irradiation per standard protocol.

It was reported by Dijkema et al. [8] that localization of the regional LNs of the breast can be dependent on the position of the arm. In order to compare LN coverage by planning contours in different treatment positions, we performed CT simulation of the reference patient in treatment position with $130^{\circ}$ abducted left arm and $160^{\circ}$ overhead abducted right arm.

Atlas creation and statistical analysis of the SLN overlap data was performed using MIM Maestro 6.9.7 software (MIM Software Inc., Cleveland, $\mathrm{OH}, \mathrm{USA}$ ). The atlas was created using the approach similar to the one described by Borm et al. [9].

First, the images were masked outside of the regions of interest corresponding to a $5-\mathrm{cm}$ margin around to reference areas. Initial rigid registration of the masked images was followed by a contour-based deformable registration using a hybrid fusion mode [10] provided by MIM Maestro software. MIM Maestro Reg Refine tool was used to assess the quality of deformable registration and to enable user-guided deformable registration adjustment as necessary [11]. Next, each SLN contour on the patient image was deformable transferred to the reference patient CT image. All steps were automated using MIM Maestro Workflows to standardize and speed up image processing for each patient. Following the atlas creation, a statistical overlap (occurrence of the SLN contours inside CTVs) was calculated using a custom MIM Maestro Extension written in Java programming language, with the overlap statistical data being exported into Excel Software (Microsoft, Redmond, WA, USA) for subsequent verification and analysis. The CTVs for LN irradiation were delineated by two experienced radiation oncologists with a cross-evaluation of the contours and a subsequent control by the third investigator. All contours were delineated according to ESTRO or RTPOG guidelines $[4,5]$. All CTVs were created before the fusion by radiation oncologists that were blinded to the locations of SLNs. The contoured subregions corresponding to ESTRO and RTOG guidelines were as follows: axillary level I, II, and III LNs, the supraclavicular, the internal mammary, interpectoral and subpectoral region. In addition, we created a separate "posterior neck volume" which was represented by the differences in RADCOMP and ESTRO/RTOG volumes.

Finally, we calculated the overlap of mapped SLNs with "standard" LNs CTV contours and assessed whether they were located within/partly within (10\%-100\% overlap) or outside (<10\% overlap) the ESTRO/RTOG and RADCOMP CTVs.

\section{Results}

In 254 women included in the study, we visualized 532 SLNs in average 2.1 SLNs per patient. Atlas of the mapped SLNs in relation to the ESTRO and RTOG CTV-LN can be found in Supplementary Figs. S1 and S2. The distribution of SLNs according to LN levels is summarized in Table 1. As was expected, most SLNs were detected in the axillary level I region ( $\mathrm{n}=359 ; 67.5 \%$ ), followed by axillary II ( $\mathrm{n}$ $=82 ; 15.5 \%)$, axillary III $(\mathrm{n}=39 ; 7.3 \%)$, the internal mammary $(\mathrm{n}$ $=45 ; 8.4 \%)$, and supraclavicular $(n=7 ; 1.3 \%)$ regions. Mapped LNs in apical (level III) and supraclavicular regions probably must be considered as second echelon LNs that are directly connected 
Table 1. Distribution of SLNs in the lymph node regions and coverage of these lymph nodes by ESTRO and RTOG contours

\begin{tabular}{|c|c|c|c|c|c|c|}
\hline \multirow{2}{*}{ Localization } & \multirow{2}{*}{ SLNs } & \multirow{2}{*}{ Number of patients } & \multicolumn{2}{|c|}{ Number of patient with SLNs inside contours } & \multicolumn{2}{|c|}{ Number of patients with SLNs outside contours } \\
\hline & & & ESTRO & RTOG & ESTRO & RTOG \\
\hline Level I & 359 & $243(100)$ & $201(82.7)$ & $207(85.2)$ & $42(17.3)$ & $36(14.8)$ \\
\hline Level II & 82 & $76(100)$ & $76(100)$ & $76(100)$ & - & - \\
\hline Level III & 39 & 39 (100) & 35 (89.7) & $36(92.3)$ & $4(10.3)$ & $3(7.7)$ \\
\hline Supraclavicular & 7 & 6 (83.3) & $5(100)$ & $6(100)$ & $1(16.4)$ & - \\
\hline Internal mammary & 45 & $33(100)$ & 30 (90.9) & $27(81.8)$ & $3(9.1)$ & 5 (18.2) \\
\hline
\end{tabular}

Values are presented as number (\%).

SLN, sentinel lymph node; ESTRO, European Society for Therapeutic Radiology and Oncology; RTOG, Radiation Therapy Oncology Group.

Table 2. Distribution of SLNs in the axillary lymph node subgroups and coverage of these lymph nodes by ESTRO and RTOG contours in women with different arm positions

\begin{tabular}{|c|c|c|c|c|c|c|c|c|c|c|}
\hline \multirow{3}{*}{$\begin{array}{l}\text { Axillary } \\
\text { subgroups }\end{array}$} & \multirow{3}{*}{ SLNs } & \multirow{3}{*}{$\begin{array}{l}\text { Number of } \\
\text { patients }\end{array}$} & \multicolumn{4}{|c|}{ Number of patient with SLNs inside contours } & \multicolumn{4}{|c|}{ Number of patient with SLNs outside contours } \\
\hline & & & \multicolumn{2}{|c|}{$\begin{array}{c}\text { Left breast } \\
\left(130^{\circ} \text { abducted }\right)\end{array}$} & \multicolumn{2}{|c|}{$\begin{array}{c}\text { Right breast } \\
\left(160^{\circ} \text { abducted }\right)\end{array}$} & \multicolumn{2}{|c|}{$\begin{array}{c}\text { Left breast } \\
\left(130^{\circ} \text { abducted }\right)\end{array}$} & \multicolumn{2}{|c|}{$\begin{array}{c}\text { Right breast } \\
\left(160^{\circ} \text { abducted }\right)\end{array}$} \\
\hline & & & ESTRO & RTOG & ESTRO & RTOG & ESTRO & RTOG & ESTRO & RTOG \\
\hline $\begin{array}{l}\text { Anterior } \\
\text { pectoral }\end{array}$ & 135 & 128 & 73 & 73 & 53 & 53 & 2 & 2 & - & - \\
\hline Central & 171 & 168 & 74 & 77 & 69 & 68 & 3 & - & 4 & 5 \\
\hline Lateral & 49 & 47 & 2 & 4 & 8 & 16 & 25 & 23 & 12 & 4 \\
\hline Subscapular & 4 & 3 & - & - & 1 & 2 & - & - & 2 & 1 \\
\hline
\end{tabular}

SLN, sentinel lymph node; ESTRO, European Society for Therapeutic Radiology and Oncology; RTOG, Radiation Therapy Oncology Group.

with axillary SLNs. This proposal is supported by the fact that in all cases axillary SLNs were detected before "hot" LNs of apical and supraclavicular regions. Distribution of the LNs that were mapped with radiocolloids according to axillary (level I) subgroups is listed in Table 2. SLNs were located in the central axillary subgroup in 171, anterior pectoral in 135, lateral in 49, and subscapular in 4 cases. The distribution of mapped LNs in patients did not differ between right and left breast cancer.

\section{Level I lymph nodes}

SLNs detected in the axillary level I were outside the ESTRO guideline borders in 42 of 243 patients with mapped level I LNs and outside RTOG LNs CTV in 36 of 243 cases. Surprisingly, we found that SLNs were frequently localized in the lateral group of axillary LNs (Fig. 1): in 47 (19.3\%) women SLNs were revealed in the lateral group, and in 14 cases SLNs were detected only in the lateral group. It must be pointed out that in many cases SLNs of the lateral group were localized out of the ESTRO and RTOG contours: $37 / 47$ and 27/47 observations, respectively. This is due to the position of the arm that significantly influences the rate of "in contours" and "out of contours" localization of the lateral group SLNs. In 119 women with the right breast cancer ( $160^{\circ}$ abduction), 12 of 20 SLNs in the lateral group were inside ESTRO and 16 of 20 inside RTOG contours. With $130^{\circ}$ abduction (left breast), lateral group SLNs were visualized "out of ESTRO contours" in 25 of 27 cases and out of RTOG borders in 12 of 27 patients.

As it was mentioned above, in most cases level I axillary SLNs are represented by anterior pectoral and the central LNs. These SLNs are usually successfully covered by LNs CTV recommended by ESTRO ( $\mathrm{n}=299$ of 306 visualized SLNs, 97.7\%) and by RTOG ( $\mathrm{n}=$ 302 of 306 visualized SLNs, 98.7\%). SLNs of central subgroup were not covered by ESTRO CTV in seven women: $1 \mathrm{~mm}$ anterior and 1 $\mathrm{mm}, 5 \mathrm{~mm}, 9 \mathrm{~mm}, 9 \mathrm{~mm}, 11 \mathrm{~mm}, 11 \mathrm{~mm}$ caudally from the ESTRO border. Central SLNs were out of RTOG contours in five cases: 1 $\mathrm{mm}, 4 \mathrm{~mm}, 4 \mathrm{~mm}, 9 \mathrm{~mm}$, and $10 \mathrm{~mm}$ caudally from the RTOG border. Anterior pectorals SLNs were $1 \mathrm{~mm}$ and $2 \mathrm{~mm}$ anterior-laterally from ESTRO CTV contour in two women and $2 \mathrm{~mm}$ anterior to RTOG CTV in one observation. We did not find any correlation between the position of the arm and the number of "out of contours" anterior pectoral and central LNs.

In the case of tangential irradiation, this "anterior shift" of the LNs CTV can potentially reduce the absorbed doses in the heart, coronary vessels (left breast), and lung but this proposal of course needs further evaluation.

We compared the coverage of the SLNs by ESTRO and RTOG contours in patients with a different arm position (left breast vs. right breast). The only and important finding was much better coverage of SLNs localized in the group of lateral axillary LNs: with $160^{\circ}$ abducted arm (right breast), they were covered by ESTRO and RTOG contours in $8(40 \%)$ of 20 and $16(80 \%)$ of 20 , respectively. 

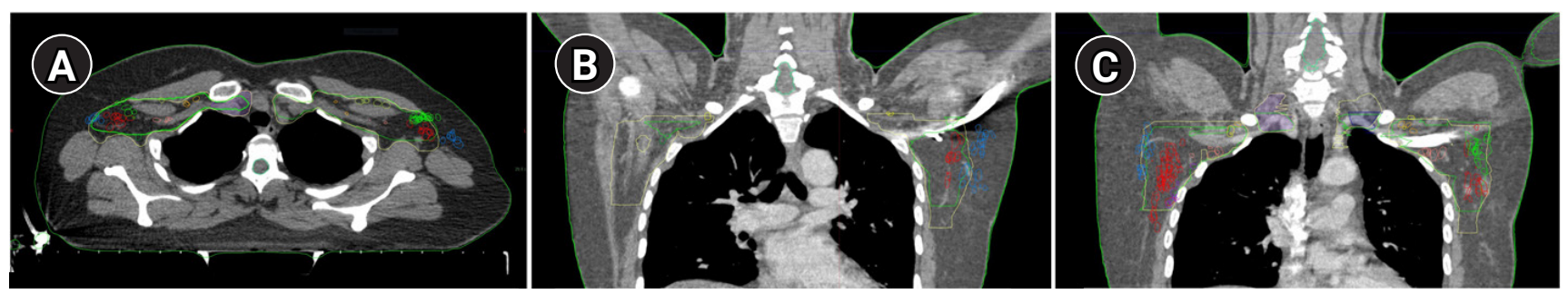

Fig. 1. Lateral subgroup of axillary lymph nodes (blue) that are mostly out and partly inside the RTOG and the ESTRO contours. (A) Axial image represent lateral (blue), central (red), and anterior pectoral (green) subgroups of axillary lymph nodes in relation to ESTRO contours (green) and RTOG contours (yellow). (B) Coronal image of left lateral axillary lymph nodes. (C) Coronal image of right lateral axillary lymph nodes. ESRTO, European Society for Radiation Therapy and Oncology; RTOG, Radiation Therapy Oncology Group.

On the contrary, in patients with $130^{\circ}$ abduction (left breast), SLNs localized in the lateral axillary group were inside of RTOG and ESTRO volumes only in $2(7.4 \%)$ of 27 and $4(14.8 \%)$ of 27 cases.

\section{Level II and III lymph nodes}

SLNs were localized on axillary level II in 71 patients, and in all cases they were successfully covered by ESTRO and RTOG contours. Subpectoral SLNs were usually localized very close (Supplementary Fig. S3) to the thoracic vault (48 observations). This fact underlines the necessity to cover the anterior surface of the I-III ribs carefully.

"Hot" LNs with uptake of radiocolloids on axillary level III and supraclavicular LNs were visualized in 39 and 7 patients. In all cases, radioactive LNs in these regions were accompanied by SLNs in level I and/or II. Apical "hot" LNs were outside the ESTRO and RTOG contours in four and three women: $1 \mathrm{~mm}, 1 \mathrm{~mm}, 8 \mathrm{~mm}, 9 \mathrm{~mm}$ cranially and $3 \mathrm{~mm}, 3 \mathrm{~mm}, 4 \mathrm{~mm}$ cranially, respectively.

\section{Supraclavicular lymph nodes}

The topography of SLNs in supraclavicular region can be important for better understanding the minimal and sufficient borders of supraclavicular CTV. First of all, we evaluated the guidelines of RADCOMP trial that recommend irradiation of posterior neck region. According to our data there were no SLNs in posterior neck in any of our six patients. Secondly, we tried to evaluate the most medial location of SLNs in supraclavicular region and found out that there was only one SLN medial to vein and we did not see any SLN medially to the common carotid artery (Fig. 2). Only one "out of ESTRO contours" SLN was visualized $1 \mathrm{~mm}$ caudally to the upper border of the supraclavicular contours but was inside RTOG contours.

\section{Internal mammary lymph nodes}

SLNs in the internal mammary region were revealed in $33(13 \%)$ of 254 evaluated patients: eight on the right side and 25 on the left. In all cases they were detected between the I rib and the IV rib. Only three internal mammary SLNs were out of ESTRO contour: 1 $\mathrm{mm}$ anterior, $1 \mathrm{~mm}$ and $2 \mathrm{~mm}$ laterally from CTV border. Internal

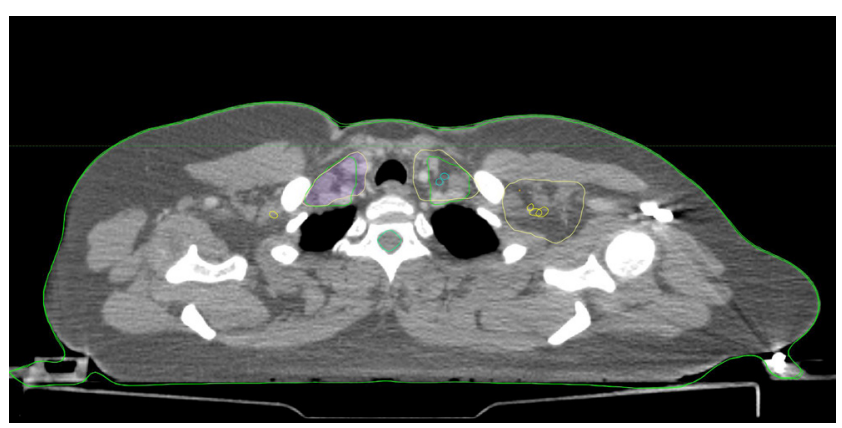

Fig. 2. Localization of the supraclavicular lymph nodes (blue) with uptake of radiocolloids with regard to the European Society for Radiation Therapy and Oncology contours (green) and the Radiation Therapy Oncology Group contours (yellow).

mammary LNs were out of RTOG contour in six cases: $1 \mathrm{~mm}, 1 \mathrm{~mm}$, $2 \mathrm{~mm}$ anterior-medially, $2 \mathrm{~mm}$ anterior-laterally, and $1 \mathrm{~mm}, 2 \mathrm{~mm}$ laterally. Obtained data indicate that $7 \mathrm{~mm}$ margins around internal mammary vessels would help to cover internal mammary SLNs in all cases.

\section{Discussion and Conclusion}

For our knowledge this is the first attempt to evaluate different international guidelines for regional LN delineation by mapping SLNs topography in large population of patients with breast cancer. A detailed SLN atlas was created using SPECT-CT data of 254 women with an early breast cancer and a non-rigid registration of these data based on vessels and muscles anatomy. An important finding of this study is the variability of lymphatic drainage from breast lesions that manifested by various localization of radioactive LNs represented by sentinel LNs and second echelon LNs which were the next step of the regional metastatic pathway. According to our data $82.9 \%$ of SLNs were visualized in axillary level I and II. This is in accordance with existing PET-CT data indicating that the majority of metastatic lymph nodes in primary patients with breast cancer are located in the axilla (91.6\%) [9]. According to Kowalski et al. [12] in patients with advanced and recurrent disease regional 
metastases detected in the axillary LNs in 73\% of all cases and in other $27 \%$ occupied internal mammary and supraclavicular regions.

Our results surprisingly demonstrated that only $82 \%-85 \%$ of level I LNs were covered by contours created according to ESTRO and RTOG guidelines. In most cases, "out of contour" LNs represented by SLNs were localized in the lateral axillary subgroup (37/42 in ESTRO and 27/36 in RTOG). Generally, these LNs are not considered as regional LNs receiving the lymph flow from the breast but represent the nodes that collect lymph flow from the arm and hence, are not the target for regional LN irradiation [13]. It must be pointed out that in studies that evaluate the pattern of regional recurrences in patient with breast cancer these nodes are rarely pointed out as "the risk area." On the other hand, the low frequency of regional recurrences in lateral axillary LNs can be explained by the high therapeutic dose that they absorb during the tangential irradiation of the breast [7]. The tendency to more complex and accurate methods of the dose delivery to the breast and LNs CTV (proton therapy, intensity-modulated radiotherapy) can be associated with a substantial underdose of lateral axillary LNs and a subsequent increased frequency of regional recurrences in this area [14]. It seems that in these cases lateral lymph nodes must be included in the LN-CTV. According to our personal experience, the irradiation in the position of $160^{\circ}$ overhead abducted right arm with 1-2 cm lateral expansion of level I LN-CTV can help to cover lateral axillary LNs in most cases.

In addition, we mentioned one regularity that could be important for radiotherapy planning: if in women with $130^{\circ}$ abducted arm (left breast) we would draw imaginary lines connecting the anterior-lateral surface of the II-V ribs with anterior surface of latissimus dorsi muscle (Fig. 3) all central, anterior-pectoral and lateral SLNs would be localized anterior to this line. The same pattern was detected in patients with $160^{\circ}$ overhead abducted arm with the imaginary line passing nearly horizontally.

It is well-known that in women with breast cancer radiotherapy significantly increases the risk of late complications. First of all, morbidity and mortally associated with cardiac disease, in particular it was demonstrated that cardiac mortality was strongly related to the dose absorbed by the heart $[15,16]$. Additionally, it was shown that radiotherapy could increase the incidence of primary lung, esophageal and contralateral breast cancers [17-19]. Our mapping results indicate that localization of SLNs on the axillary level I give the opportunity to move forward the posterior border of the level I contours and significantly reduce the irradiated volume and doses absorbed by heart (in left-sided breast cancer), lung and contralateral breast.

Existing studies that conducted a three-dimensional analysis of $\mathrm{LN}$ recurrences and/or topography of $\mathrm{LN}$ metastases in patients with breast cancer indicate that around 13\%-34\% of regional recurrences were located in the supraclavicular LNs $[9,19,20]$. According to the study by Chang et al. [20], supraclavicular LNs were the most frequent site of regional recurrences (33.8\%); DeSelm et al. [19] revealed supraclavicular LNs metastases in 25.5\%; and Borm et al. [9] in $13.8 \%$. According to our data, only $2.3 \%$ of evaluated patients had radioactive ("hot") LNs in supraclavicular area and as was mentioned before we believe that most of these LNs were second echelon nodes. We found out that all these LNs were located laterally to common carotid artery and caudal to cricoid cartilage. Nielsen and Offersen [21] also concluded that ESTRO contours of supraclavicular area were correct and did not need enlargement. On the contrary, many authors reported that mapping of supraclavicular recurrent or clinically involved LNs demonstrated that around $20 \%-41 \%$ of metastases in this region were located out of RTOG and/or ESTRO contours and nearly always associated with an advanced nodal disease $[9,12,20,22]$. Usually missed metastatic supraclavicular LNs were found laterally to sternocleidomastoid muscles, posterior to the transverse process of the vertebral body $[19,22,23]$ or cranial to the subclavian artery [24] mostly at the level of thyroid and cricoid cartilage [25]. Gee et al. [14] summarizing the existing studies mentioned that the most vulnerable to geographical miss were the cranial and posterolateral contours of supraclavicular area.
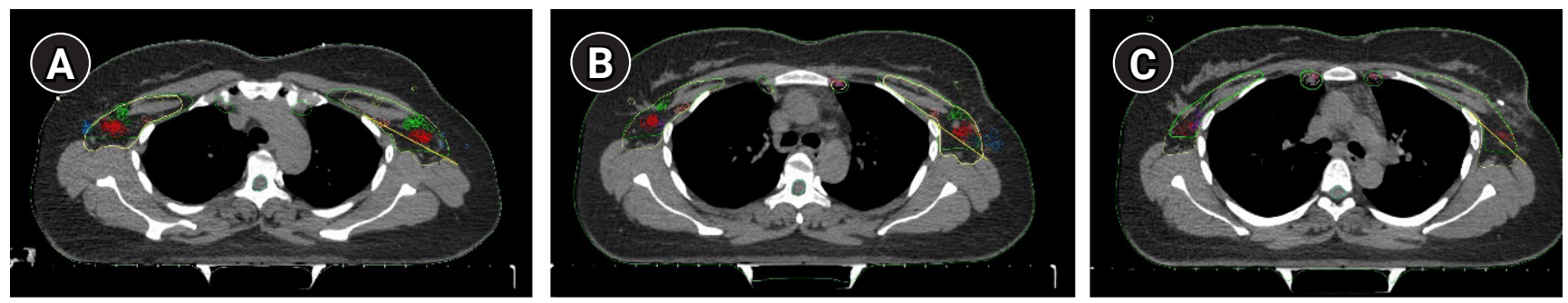

Fig. 3. Axial images of the left axillary lymph nodes. Yellow line represents the plan that connects the anterior-lateral surface of the II- $V$ ribs and anterior surface of latissimus dorsi muscle. All anterior pectoral (green) and central (red) axillary lymph nodes are visualized anterior to this plan. (A) On the level of the II rib. (B) On the level of the III rib. (C) On the level the V rib. 
Prospective randomised and observation studies, retrospective analysis of these trials showed that irradiation of internal mammary LNs could improve overall and distant metastatic free survival [26-28]. We visualized SLNs in internal mammary region in 33 $(13 \%)$ of 254 evaluated patients. All these SLNs were located cranially to the 4th rib and were successfully covered by ESTRO and RTOG contours. These data is in accordance with the previous experience that indicated that $90 \%$ of metastatic internal mammary LNs were detected in the 1st-3rd intercostals spaces $[12,24,29]$ and were successfully covered by ESTRO, RTOG, and RADCOMP contours.

Our data have several important limitations. In contrast with previous studies that mapped recurrent or FDG-positive LNs we evaluate topography of sentinel and second echelon LNs-nodes with the highest risk of involvement in patients with an early breast cancer. This means that our data must be used with caution in women with the advanced nodal disease and can be more applicable for women with 1-3 positive LNs or in case of irradiation after positive sentinel LN biopsy. It must be also mentioned that all women who underwent SPECT-CT examinations had an early breast cancer (T1-3N0-1M0).

Moreover, despite the deformable algorithm of fusion the accuracy of this procedure can be slightly compromised by a difference in arm position between SPECT-CT and simulation CT.

In conclusion, SPECT-CT atlas of SLNs demonstrated that in most cases ESTRO and RTOG guidelines were effective for contouring of axillary II-III, supraclavicular and internal mammary LNs. In 18.5\% of evaluated patients, SLNs were located in lateral axillary subgroup that in most cases was not covered by ESTRO and RTOG contours. These data must be specially considered in cases when tangential irradiation is substituted by intensity-modulated radiotherapy or proton therapy. Our data indicate that in patients with 1-3 positive LNs posterior border of level I axillary LNs can be safely shifted anteriorly (to the imaginary line connecting anterior-lateral surface of the II-V ribs with anterior surface of latissimus dorsi muscle) that would help to reduce absorbed doses in lung and heart.

\section{Conflict of Interest}

No potential conflict of interest was reported by the authors.

\section{Acknowledgements}

The authors would like to acknowledge MIM Software Inc. (Cleveland, $\mathrm{OH}, \mathrm{USA}$ ) for providing MIM Maestro software used in this study and Dr. Oleg Egorov for enabling the automation of image processing and statistical analysis.

\section{Supplementary Materials}

Supplementary materials can be found via https://doi.org/10.3857/ roj.2020.00871.

\section{References}

1. Nieweg OE. The sentinel lymph node concept. In : Mariani G, Vidal-Sicart S, Valdes Olmos RA, editors. Atlas of lymphoscintigraphy and sentinel node mapping Cham, Switzerland: Springer; 2020, p. 143-9.

2. Krag DN, Anderson SJ, Julian TB, et al. Sentinel-lymph-node resection compared with conventional axillary-lymph-node dissection in clinically node-negative patients with breast cancer: overall survival findings from the NSABP B-32 randomised phase 3 trial. Lancet Oncol 2010;11:927-33.

3. Mansel RE, Fallowfield L, Kissin M, et al. Randomized multicenter trial of sentinel node biopsy versus standard axillary treatment in operable breast cancer: the ALMANAC Trial. J Natl Cancer Inst 2006;98:599-609.

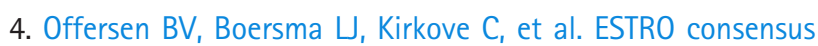
guideline on target volume delineation for elective radiation therapy of early stage breast cancer, version 1.1. Radiother Oncol 2016;118:205-8.

5. White J, Tai A, Arthur D, et al. Breast cancer atlas for radiation therapy planning: consensus definitions [Internet]. Philadelphia, PA: Radiation Therapy Oncology Group; 2018 [cited 2021 Feb 20]. Available from: https://www.nrgoncology.org/Portals/0/Scientific\%20Program/CIRO/Atlases/BreastCancerAtlas_corr.pdf? ver $=$ 2018-04-18-144201-270.

6. NRG Oncology. RADCOMP breast atlas [Internet]. Philadelphia, PA: NRG Oncology; c2019 [cited 2021 Feb 20]. Available from: https://www.nrgoncology.org/About-Us/Center-for-Innovation-in-Radiation-Oncology/Breast/RADCOMP-Breast-Atlas.

7. Novikov S, Krzhivitskii P, Kanaev S, et al. SPECT-CT localization of axillary sentinel lymph nodes for radiotherapy of early breast cancer. Rep Pract Oncol Radiother 2019;24:688-94.

8. Dijkema IM, Hofman P, Raaijmakers CP, Lagendijk JJ, Battermann $J$ J, Hillen B. Loco-regional conformal radiotherapy of the breast: delineation of the regional lymph node clinical target volumes in treatment position. Radiother Oncol 2004;71:287-95.

9. Borm KJ, Voppichler J, Dusberg M, et al. FDG/PET-CT-based lymph node atlas in breast cancer patients. Int J Radiat Oncol Biol Phys 2019;103:574-82.

10. Takayama Y, Kadoya N, Yamamoto T, et al. Evaluation of the per- 
formance of deformable image registration between planning CT and $\mathrm{CBCT}$ images for the pelvic region: comparison between hybrid and intensity-based DIR. J Radiat Res 2017;58:567-71.

11. Johnson PB, Padgett KR, Chen KL, Dogan N. Evaluation of the tool "Reg Refine" for user-guided deformable image registration. J Appl Clin Med Phys 2016;17:158-70.

12. Kowalski ES, Feigenberg SJ, Cohen J, et al. Optimal target delineation and treatment techniques in the era of conformal photon and proton breast and regional nodal irradiation. Pract Radiat Oncol 2020;10:174-82.

13. Wang $W$, Ward $R$, Jia $D$, et al. Location of arm draining lymph node in relation to breast cancer radiotherapy field and target volume. Radiother Oncol 2019;133:193-7.

14. Gee HE, Moses L, Stuart K, et al. Contouring consensus guidelines in breast cancer radiotherapy: comparison and systematic review of patterns of failure. J Med Imaging Radiat Oncol 2019;63:10215.

15. Early Breast Cancer Trialists' Collaborative Group (EBCTCG). Effect of radiotherapy after breast-conserving surgery on 10-year recurrence and 15-year breast cancer death: meta-analysis of individual patient data for 10,801 women in 17 randomised trials. Lancet $2011 ; 378: 1707-16$.

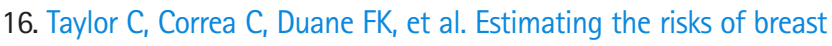
cancer radiotherapy: evidence from modern radiation doses to the lungs and heart and from previous randomized trials. J Clin Oncol 2017;35:1641-9.

17. Grantzau T, Overgaard J. Risk of second non-breast cancer after radiotherapy for breast cancer: a systematic review and meta-analysis of 762,468 patients. Radiother Oncol 2015;114:5665.

18. Morton LM, Gilbert ES, Hall P, et al. Risk of treatment-related esophageal cancer among breast cancer survivors. Ann Oncol 2012;23:3081-91.

19. DeSelm CJ, Yang TJ, Tisnado J, et al. Regional patterns of breast cancer failure after definitive therapy: a large, single-institution analysis. Int J Radiat Oncol Biol Phys 2016;96:S145.

20. Chang JS, Byun HK, Kim JW, et al. Three-dimensional analysis of patterns of locoregional recurrence after treatment in breast cancer patients: Validation of the ESTRO consensus guideline on target volume. Radiother Oncol 2017;122:24-9.

21. Nielsen HM, Offersen BV. Regional recurrence after adjuvant breast cancer radiotherapy is not due to insufficient target coverage. Radiother Oncol 2015;114:1-2.

22. Reed VK, Cavalcanti JL, Strom EA, et al. Risk of subclinical micrometastatic disease in the supraclavicular nodal bed according to the anatomic distribution in patients with advanced breast cancer. Int J Radiat Oncol Biol Phys 2008;71:435-40.

23. Jing $H$, Wang SL, Li J, et al. Mapping patterns of ipsilateral supraclavicular nodal metastases in breast cancer: rethinking the clinical target volume for high-risk patients. Int J Radiat Oncol Biol Phys 2015;93:268-76.

24. Chang JS, Lee J, Chun M, et al. Mapping patterns of locoregional recurrence following contemporary treatment with radiation therapy for breast cancer: a multi-institutional validation study of the ESTRO consensus guideline on clinical target volume. Radiother Oncol 2018;126:139-47.

25. Brown LC, Diehn FE, Boughey JC, et al. Delineation of supraclavicular target volumes in breast cancer radiation therapy. Int J Radiat Oncol Biol Phys 2015;92:642-9.

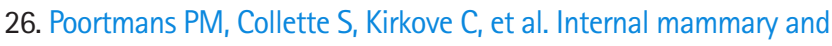
medial supraclavicular irradiation in breast cancer. N Engl J Med 2015;373:317-27.

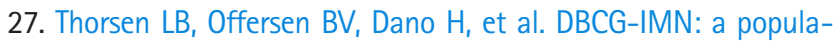
tion-based cohort study on the effect of internal mammary node irradiation in early node-positive breast cancer. J Clin Oncol 2016:34:314-20.

28. Nikolaevich NS, Vasilevich KS. Why do we need irradiation of internal mammary lymph nodes in patients with breast cancer: analysis of lymph flow and radiotherapy studies. Rep Pract Oncol Radiother 2017;22:37-41.

29. Jethwa KR, Kahila MM, Hunt KN, et al. Delineation of internal mammary nodal target volumes in breast cancer radiation therapy. Int J Radiat Oncol Biol Phys 2017;97:762-9. 